International Journal of Engineering \& Technology, $7(4.7)(2018) 136-140$
International Journal of Engineering \& Technology
SPC
Website: www.sciencepubco.com/index.php/IJET
Research paper

\title{
Angle of Arrival Estimation in Smart Antenna for Wireless Communication
}

\author{
T. S. Jyothi Lakshmi ${ }^{1 *}$, S. Sandeep ${ }^{2}$, Dr. V. Rajya Lakshmi ${ }^{3}$ \\ ${ }^{1}$ Research Scholar, Dept. of E.C.E, A.U. College of Engineering (A) \\ ${ }^{2}$ Assistant Professor, Dept. of E.C.E, School of Engineering, G.V.P. College for Degree and P.G Courses \\ ${ }^{3}$ Dr. V. Rajya Lakshmi, Professor, Dept. of E.C.E, Anil Neerukonda Institute of Technology \& Sceinces \\ *Corresponding author E-mail: jyoths.lakshmi@gmail.com
}

\begin{abstract}
Smart antenna technology has emerged as one of the most efficient techniques in supporting maximum communication link throughput. A smart antenna system with innovative signal processing can enhance the resolution of a signal's direction of arrival estimation. Successful design of Smart antenna is dependent on the efficient performance of DOA estimation algorithm as well as beamforming algorithm. This paper presents a comparative performance study between Classical Beam forming method, MUSIC (Multiple Signal Classification), MVDR (Minimum Variance Distortion less Response) which are different direction of arrival algorithms used in linear antenna array. The objective is to analyse and compare these algorithms and determine which provides maximum efficiency.
\end{abstract}

Keywords: Use about five key words or phrases in alphabetical order, Separated by Semicolon.

\section{Introduction}

For any communication system maximum throughput is the most desirable factor. The two primary criteria for Smart antennas are position estimation. Basically the processing of the signals are done then to find the location of the source. The elevation and azimuthal angles represent the position of the source in radial coordinate system [1-4]. The major lobe of the radiation beam pattern will be directed towards the estimated Angle of Arrival. Thus the maximum power which will be emitted by the antenna will be directed towards the required source which can result in that direction a very high antenna gain. The advantages of Smart antenna array are mainly the increased capacity from existing cell sites, efficient networks and also improvement in the performance. The purpose of this paper is to investigate the use of smart antennas in the cellular telecommunication network. In this paper, after introducing the antenna and examining its role in telecommunication systems, multiple access methods in the telecommunication network have been addressed. The topic of the smart antenna is discussed and intelligent antenna background, smart antenna technology and all kinds of smart antennas are spoken. The topic of the smart antenna is discussed and intelligent antenna background smart antenna technology and all kinds of smart antennas are spoken. Today, the efforts of followers are being used to make more use of waves instead of wires in the world of communications. This has several advantages: first, the use of wireless communication methods instead of wired methods, requires us to use the massive amount of wires needed.

\section{Doa Conventional Methods}

Two methods for determining the Direction of Arrival which are conventional methods. This has several advantages: first, the use of wireless communication methods instead of wired methods, requires us to use the massive amount of wires needed. Another feature, which seems more important, is the ability to switch users, that is, to communicate in a mobile way. That is, the user can use the service while moving or in any place that is covered by the antenna coverage.

\section{Classical Beamforming Method/ Bartlett Method}

Other name for Classical Beamforming Method (CBF) is the delay-and-sum method or Bartlett method [3]. If the angle in the look direction is changed in small steps across the space of interest is given by

$$
P_{C B F}(\theta)=\frac{a^{H}(\theta) R_{X X} a(\theta)}{a^{H}(\theta) a(\theta)}
$$

This quantity can be called as spatial spectrum and the angle $\theta$ at which the maximum output is obtained will be the true angle of arrival. The major drawback of this method is its poor resolution. 


\section{Capon's Minimum Variance}

The Capon's minimum variance approach also referred as the Minimum Variance Distortion Less Response (MVDR) is a method adopted to minimise the poor resolution problem which is the weakness of the delay-and-sum method and it always provided much better results. The output power is reduced here with a limitation that the gain in the required direction is kept as unity. By keeping this restriction, we solve for optimising the output, the weight vectors can be obtained as below.

$$
w=\frac{R_{X X}{ }^{-1} a(\theta)}{a^{H}(\theta) R_{X X}^{-1} a(\theta)}
$$

From this relation Capon's Spatial Spectrum can be determined as

$$
P_{\text {Capon }}(\theta)=w^{H} R_{X X} w=\frac{1}{a^{H}(\theta) R_{X X}{ }^{-1} a(\theta)}
$$

The MVDR beam former will show a considerable improvement over Bartlett method for a particular angle of arrival because it is able to reduce the power from unwanted directions. It can be seen that in MVDR the auto covariance matrix is inverted compared to the CBF and is found to give better resolution in most cases [4]. The advantage of MVDR is that it can use different range of angles $\theta$ with the same set of data for determining the spatial spectrum. The auto covariance matrix $R_{x x}$ which is to be calculated only once represents the complete spatial characteristics of the data for all directions. Because of this, MVDR method will not have blind spots when temporary passing signals which are away from the actual transmitting sources appear in between. In smart antenna, by steering electronically the speed of the surveillance is limited only by the computational speed.

\section{Doa Subspace Method}

Subspace methods are the next type of DOA estimation algorithms. In this technique the received signal space is divided into two separate spaces. One is called as the signal subspace and the other one is noise subspace. The subspace which will be spanned by the columns of the array response matrix $\mathrm{A}(\Theta)$ will be the signal subspace and noise subspace will be orthogonal to the signal subspace. The DOA of the signal can be calculated by these subspace algorithms by working on this orthogonality. MUSIC (Multiple Signal Classification) and ESPRIT (Estimation of Signal Parameters via Rotational Invariant Techniques) are the two most important type of subspace algorithms which are widely studied by most of the researchers. The error calculated while measuring the angle of arrival by ESPRIT algorithm is much higher when compared with MUSIC Hence a study is done on MUSIC only here.

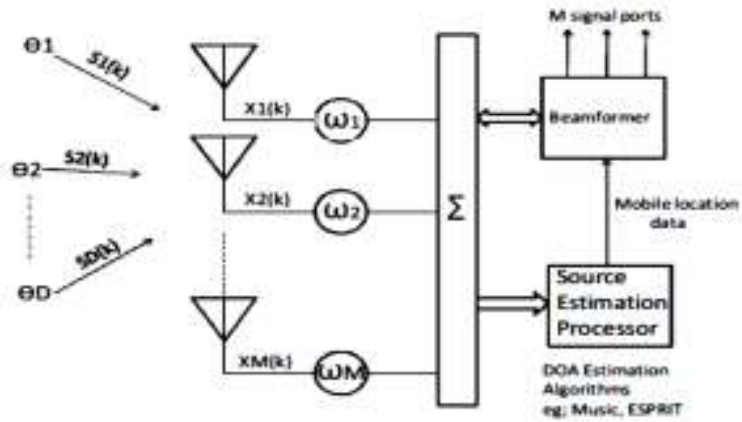

Fig 1:. DOA estimation methods

\section{The Music Algorithm}

MUSIC Algorithm was conceptualised by Schimdt and it revolutionised the idea of estimation of angle of arrival. It could deal with any arbitrary linear array configurations as there were no constraints in designing the array. This algorithm went through many modifications. The Eigen structure of the input covariance matrix is exploited in Multiple Signal Classification(MUSIC) technique. This algorithm gives also information regarding DOA of each signal, the number of incident signals, and also cross correlations between incident signals. MUSIC algorithm became very popular due to its generality. But the drawback here is array response matrix has to be calculated for all different combinations of sources and should be saved [4]. The different parameters from each source like elevation, azimuth, range etc., can also be determined using this algorithm. But it needs prior information about interference, back ground noise etc., which are second order spatial characteristics [5-7]

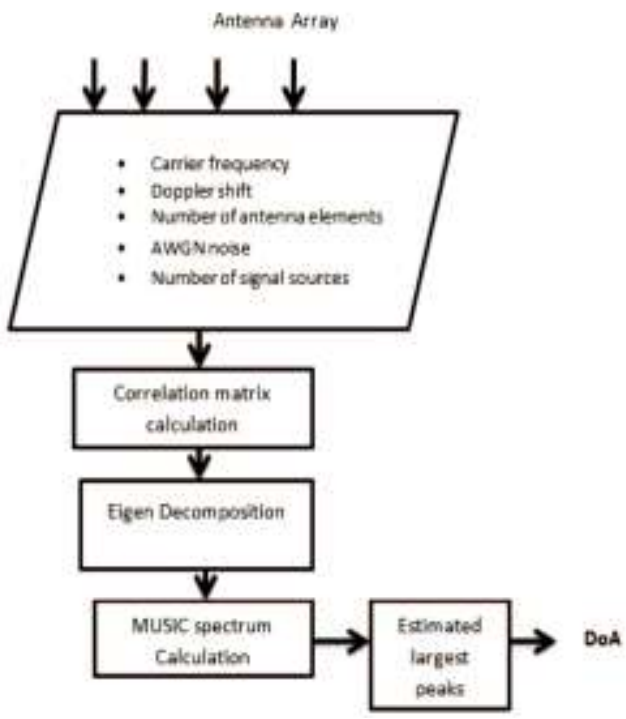

Fig 2:. DOA using MUSIC Algorithm

\subsection{MUSIC Spatial Spectrum}

The general array manifold is defined to be the set in the algorithm

$A=\left\{a\left(\theta_{i}\right): \theta_{i} \in \Theta\right\}$

That is, the user can use the service while moving or in any place that is covered by the antenna coverage.

Some of these efforts have come to a good end, but some are still in the process of testing and research. Satellite communications via receiving and sending antennas are one of the most prominent and highly effective examples in this area, which is now commonly used. Services such as cellular networks, wireless local area networks, Wi-Fi, Bluetooth, and similar services are the same. However, more advanced techniques are in place that this time, they are targeting another part of the wireless network, and that is not just antennas. The technological advances have led us to use hardware and software techniques to design a variety of antennas that have a better performance than the past. Continue with Thousands. $\mathrm{R}_{\mathrm{xx}}$ can be calculated as:

$$
\begin{aligned}
& R_{x x}=\sum_{n=1}^{N} \lambda_{n} e_{n} e_{n}^{H}=E \wedge e^{H}=E_{s} \wedge_{s} E_{s}^{H}+E_{n} \wedge_{n} \\
& =E_{s} \wedge_{s} E_{s}^{H}+\sigma_{n}^{2} E_{n} E_{n}^{H}=E_{s} \wedge_{s} E_{s}^{H}+\sigma_{n}^{2} I
\end{aligned}
$$


where

$$
\begin{aligned}
& E=\left[e_{1}, e_{2}, \ldots, e_{N}\right], E_{s}=\left[e_{1}, e_{2}, \ldots, e_{K}\right], \\
& E_{n}=\left[e_{K+1}, e_{K+2}, \ldots, e_{N}\right], \\
& \wedge=\operatorname{diag}\left\{\lambda_{1}, \lambda_{2}, \ldots, \lambda_{N}\right\}, \wedge_{S}=\operatorname{diag}\left\{\lambda_{1}, \lambda_{2}, \ldots, \lambda_{K}\right\}, \\
& \wedge_{n}=\operatorname{diag}\left\{\lambda_{K+1}, \lambda_{K+2}, \ldots, \lambda_{N}\right\}, \wedge_{s}=\wedge-\sigma_{n}^{2} I
\end{aligned}
$$

The MUSIC spatial spectrum is calculated over the region of interest as below after determining the subspaces.

$$
P_{\text {MUSIC }}=\frac{a^{H}(\theta) a(\theta)}{a^{H}(\theta) E_{n} E_{n}^{H} a(\theta)}
$$

The angle of arrival of the user signals can be calculated from the MUSIC spectrum. While deriving $\mathrm{R}_{\mathrm{xx}}$ due to lot of approximations, the noise subspace eigenvalues will not be always equal to $\sigma_{\mathrm{n}}{ }^{2}$. But it will form a cluster around this value and can be differentiated from the Eigen values of signal subspace. MUSIC gives perfect parameter estimates for multiple incident wave fronts, because both $R_{s s}$ and $E_{n}$ are always measured perfectly. The advantages of MUSIC are innumerable, but the main drawback is that the computational cost is very high and it also requires a large storage capacity for the entire array calibration data [7]. Another disadvantage of MUSIC is that if the SNR is very low or the sources are found to be very close to each other, multiple peaks are observed. Consider an $\mathrm{M}$ element array and if the number of signals impinging is D, then there will be D signal Eigen values and Eigen vectors and M-D noise Eigen values and Eigen vectors. The array correlation matrix with uncorrelated noise is given by

$$
R_{x x}=A R_{s s} A^{H}+\sigma_{n}^{2} I
$$

can be constructed as below.

$$
V_{N}=\left[v_{1} v_{2} v_{3} \ldots v_{M-D}\right]
$$

The array steering vectors and the noise subspace eigenvectors will be orthogonal to each other at the angles of arrivals $\theta_{1}, \theta_{2}, \theta_{3}$, $\theta_{\mathrm{D}}$ and the MUSIC Pseudo spectrum will be obtained as

$$
P_{\text {MUSIC }}(\theta)=\frac{1}{a b s\left(a(\theta){ }^{H} V_{N} V_{N}^{H} a(\theta)\right)}
$$

\section{Simulation Results}

For the simulation of conventional and subspace methods a 10 element. The simulation has been done with MATLAB-2014a. The actual location of the source in azimuth can only be determined by Angle of Arrival. In MUSIC, $\mathrm{k}$ no of samples or snap shots are taken for determining $\mathrm{R}_{\mathrm{xx}}$ The sources of same frequency and uncorrelated are only considered. Simulation is done for 3 signals arriving from different angles for 10 elements and 1000 samples initially. Angles are $-25^{\circ},-5^{\circ}, 30^{\circ}$. All the plots of the algorithms have been normalised for the comparison purpose.

Fig 3 shows the Spectrum of Classical, MVDR and MUSIC algorithm when the signals are coming from all the three angles.

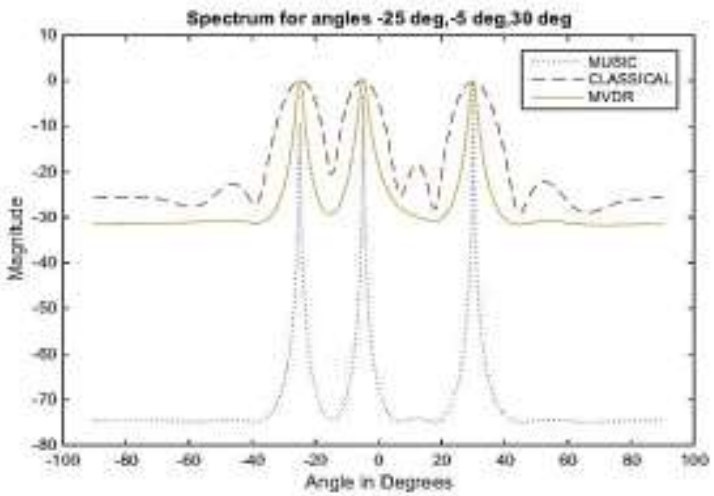

Fig 3:. DOA for MUSIC, MVDR and CLASSICAL algorithm when signal is coming from angles $-25^{\circ},-5^{\circ}, 30^{\circ}$

From the Fig 3 it is observed that MUSIC algorithm gives the best beam in the direction of users and provides nulls in the direction of interfering signals.

Fig 4 (a) and (b) shows the change in the spectrum for different number of samples for all the three algorithms.

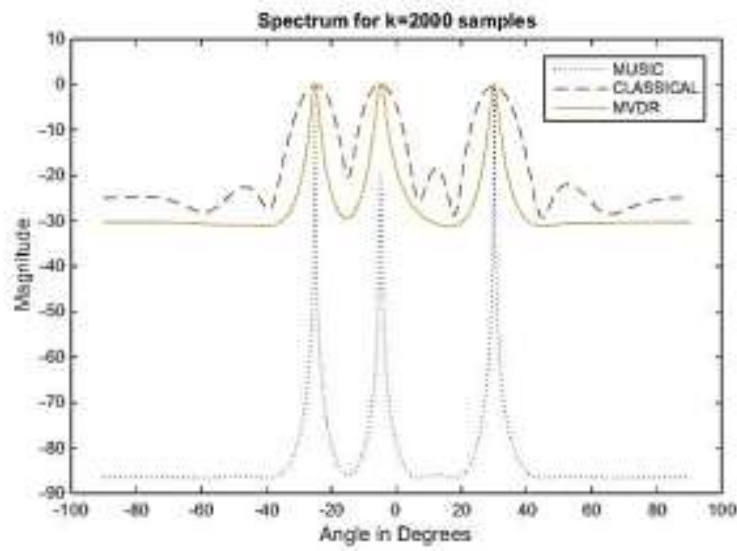

(a)

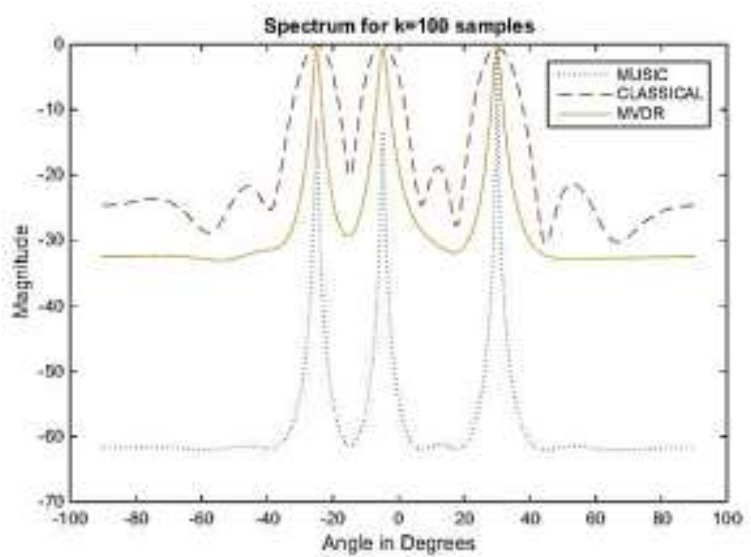

(b)

Fig 4:. DOA for the three algorithms with different number of samples a) $\mathrm{k}=2000 \mathrm{~b}) \mathrm{k}=100$

From Fig 4 (a) and (b) it can be observed that when the number of samples increased the resolution increased and it was easy to identify the signals and in the unwanted direction power dissipation also decreased.

Fig 5 (a), (b) and (c) shows the simulation of all the three algorithms for the same number of samples but with different array element spacing. 


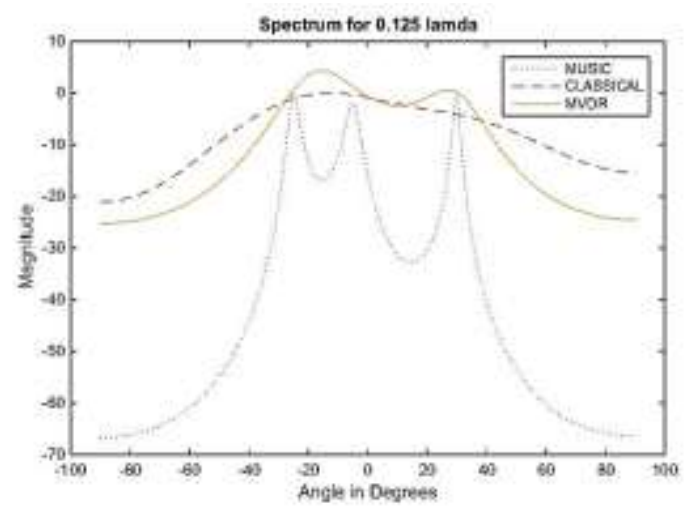

(a)

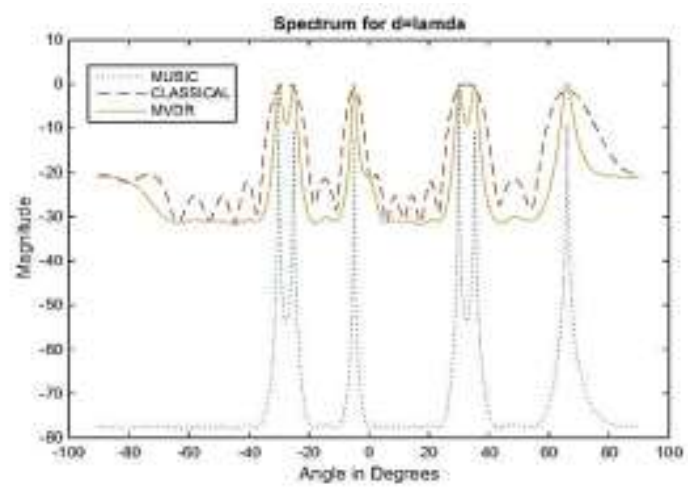

(b)

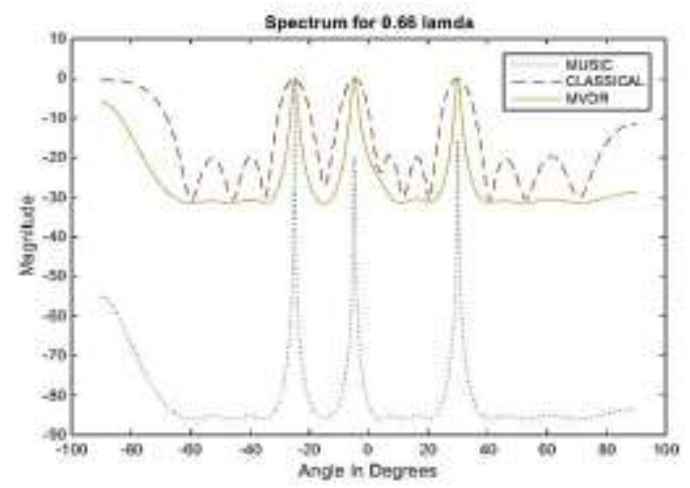

(c)

Fig 5:. DOA for different algorithms with different array element spacing $\begin{array}{lll}\text { a) } 0.125 \lambda & \text { b) } \lambda & \text { c) } 0.66 \lambda\end{array}$

From the Fig 5 it can be seen that for $d=\lambda$ and $d=0.66 \lambda$, the spectrum has additional unwanted beams. It is clearly evident that $\mathrm{d}=0.125 \lambda$, the algorithms cannot be used at all. If the spacing between the elements is equal to half the wavelength, it gives accurate results.

Fig 6 (a) and (b) shows the spectrum for different number of array elements for the three different algorithms

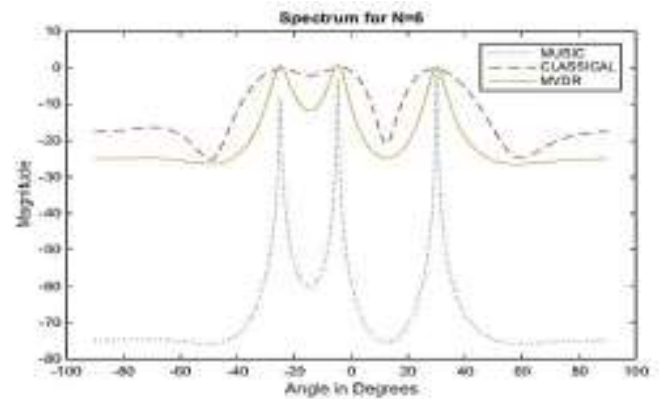

(a)

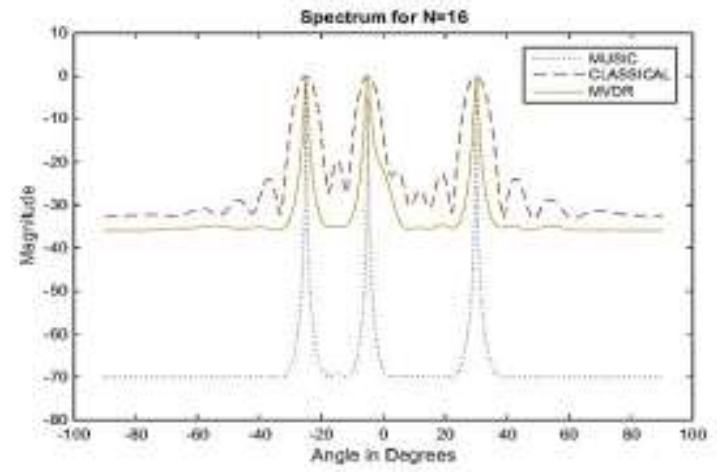

(b)

Fig 6:. DOA spectrum for different algorithms with different number of array elements a) $\mathrm{N}=6$ b) $\mathrm{N}=16$

It is seen from Fig 6 that when there was an increase in number of array elements it was found that the MVDR spectrum gave sharper peaks and MUSIC algorithm showed the best results.

Fig 7 shows the Spectrum for different DOA Algorithms for very close angles.

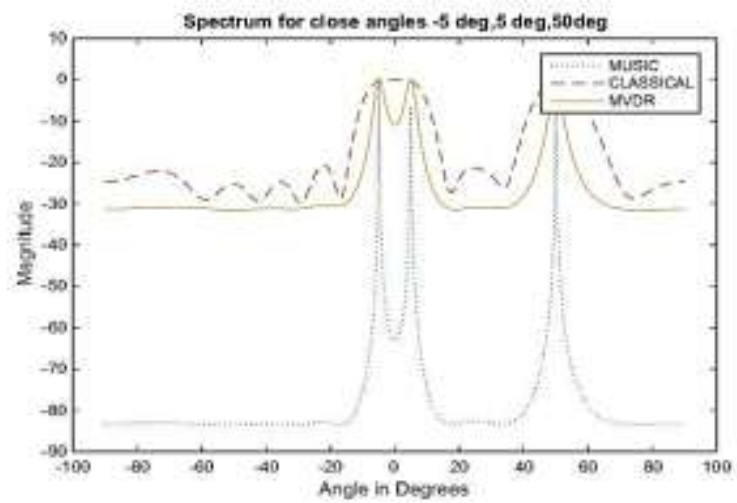

Fig 7:. DOA algorithm spectrum for close signals arriving at $-5^{\circ}, 5^{\circ}, 50^{\circ}$

It can be observed that MUSIC Algorithm is able to differentiate closely spaced signals accurately compared to other algorithms.

\section{Conclusion}

A comparative performance study of three different algorithms Classical, MVDR and MUSIC algorithms for Angle of Arrival is done in this paper. There is a drastic improvement in all the algorithms when the number of array elements are more and also for more number of snap shots. This can be observed with the help of sharper peaks in the spectrum. But it can be seen that MUSIC Algorithm showed little improvement in comparison to other two algorithms. So, if MUSIC algorithm is able to give good results with less number of array elements then it can reduce the computational time also and this is a very important parameter in embedded antenna design. It was also observed that MUSIC performed much better with less angular separation between the signals. For different value of element spacing, number of elements and samples, all the three algorithms were compared and it was observed that MUSIC is very stable and accurate algorithm as well as it gives good resolution.

\section{References}

[1] Pradhumna Lal Shrestha, Michael Hempel, Puttipong Mahasukhon, Tao Ma and Hamid "Performance Analysis for Direction of Arrival Estimating Algorithms”IEEE 2012 
[2] Mohammadzadeh, S., Zadkarim, S., Ammari H., Re-Engineering of Drinking Water Facilities of Villages of City of Saqqez for Water Loss Reduction Management, Supplement No. 2, 2017, p. 859.

[3] Popovici I., Metode interactive de predare a matematicii. Softwareul GeoGebra, Supplement No. 2, 2017, p. 875.

[4] N. Jadhav, V. M. Mhalgi, S. D. Bhosale, P.B. Khapale, "Evaluation of PM and MUSIC: Direction of Arrival Estimation for Smart Antenna System", International Journal of Engineering, Economics and Management Volume 1, Issue 1, Sep-2012.

[5] Zhizhang Chen , Gopal K. Gokeda, Yiqiang Yu , "Introduction to Direction-of-arrival Estimation", International Journal of Scientific \& Engineering Research Volume 3, Issue 7, 2010.

[6] Zhi Zheng ,Guangiun Li , "Fast DOA estimation of incoherently distributed sources by novel propagator", Journal Multidimensional Systems and Signal Processing archive Volume 24 Issue 3, September 2013.

[7] Mr. D. B. Salunke, Dr. R. S. Kawitkar, "Analysis of LMS and MUSIC Algorithms for Adaptive Array Antenna System”, International Journal of Advanced Electrical and Electronics Engineering, (IJAEEE), ISSN (Print): 2278-8948, Volume-1, Issue-3, 2012. 